\title{
Estudio de la actividad catalítica de la ferrita de cobalto en la degradación de diclofenac sódico
}

\section{Study of cobalt ferrite catalityc activity in sodium diclofenac degradation}

Presentación: 07/10/2020

\section{Doctorando:}

\section{María Verónica Gerbaldo}

Centro de Investigación y Tecnología Química, Facultad Regional Córdoba, Universidad Tecnológica Nacional, Argentina. mgerbaldo@frc.utn.edu.ar

\section{Director/a:}

\section{Mónica Elsie Crivello}

\section{Co-director/a:}

\section{Silvia Nazaret Mendieta y Verónica Rita Elías}

\section{Resumen}

Los contaminantes emergentes y dentro de ellos los compuestos farmacéuticos suelen encontrarse en aguas superficiales y residuales a lo largo del planeta. En este trabajo se presenta el estudio de la degradación de diclofenac sódico mediante un proceso tipo foto-Fenton heterogéneo empleando ferritas de cobalto. Los materiales fueron sintetizados con el método de Pechini y caracterizados mediante difracción de rayos X, espectroscopía Mössbauer, espectroscopía fotoelectrónica de rayos X, espectroscopía Uv-visible, reducción a temperatura programada y área superficial. Se obtuvieron fases puras con estructura de espinela. Ambos materiales presentaron buenos resultados catalíticos en la degradación del contaminate. Se propone un mecanismo de reacción radicalario basado en la formación de radicales ${ }^{*} \mathrm{OH}$. Se logró una degradación completa del compuesto, con un $86 \%$ de mineralización del carbono orgánico total empleando el material calcinado a $800^{\circ} \mathrm{C}$. El catalizador presentó excelentes resultados en tres ciclos consecutivos de uso.

Palabras clave: ferrita de cobalto, diclofenac, degradación

\begin{abstract}
Emerging pollutants, among them pharmaceutical compounds, are found in surface and ground waters, suggesting their ineffective removal by conventional wastewater treatment technologies. In this work, the removal of sodium diclofenac was studied by a heterogeneous photo-Fenton process using cobalt ferrites, oxygen peroxide, and Uv radiation. The catalysts were obtained by the Pechini method. They were characterized by X-ray diffraction, Uv visible spectroscopy, Mössbauer, and X-ray photoelectron spectroscopy, temperature-programmed reduction, and specific surface area. The inverse spinel structure was obtained, and both materials presented good performance in the sodium diclofenac degradation. A reaction mechanism based on the generation of ${ }^{*} \mathrm{OH}$ groups is suggested. The complete degradation and $86 \%$ of the pollutant mineralization were obtained using cobalt ferrite calcined at $800^{\circ} \mathrm{C}$. The catalyst was proved good recycling performance.
\end{abstract}




\section{Introducción}

En los últimos años, numerosas investigaciones han comenzado a hablar de los contaminantes emergentes (CE), sustancias químicas orgánicas de origen natural o sintético cuya presencia en aguas residuales no se encuentra regulada. Los CE pueden causar efectos adversos en el medio ambiente y en la salud humana [1].

Los compuestos farmacéuticos forman parte de los CE y su presencia está siendo detectada en aguas residuales a lo largo del mundo. Se han detectado en ríos, lagos y aguas subterráneas de países europeos, asiáticos y en Estados Unidos [2]. También en América Latina, Valdés et al. [3] estudiaron la presencia de fármacos en el Río Suquía, en la provincia de Córdoba. Atenolol, carbamazepina, y diclofenac fueron los más detectados en concentraciones inferiores a $1 \mu \mathrm{g} / \mathrm{L}$. a pesar de las bajas concentraciones encontradas en cursos hídricos, su presencia tiene un impacto significativo en los ecosistemas [3].

El diclofenac sódico es un antiinflamatorio no esteroideo comúnmente utilizado como analgésico para el tratamiento de la artritis y el reuma. Aproximadamente el 15\% de la ingesta humana es excretado [4]. Es un fármaco difícil de degradar en tratamientos de aguas convencionales, en general los tratamientos biológicos logran eliminar entre el 20 y $40 \%$ del compuesto [3] Los procesos avanzados de oxidación (PAOs) presentan especial interés por su potencial aplicación para el tratamiento de aguas residuales que contengan $\mathrm{CE}[5]$.

Las ferritas con estructura de espinela $\left(\mathrm{MFe}_{2} \mathrm{O}_{4}\right.$, donde $\mathrm{M}$ representa a un metal de transición) exhiben excelentes características catalíticas, estructurales y de estabilidad [6]. Existen importantes estudios sobre la aplicación de estos materiales como fotocatalizadores en la degradación de CE, presentando resultados efectivos bajo radiación con el agregado de un agente oxidante en la generación del par electron-hueco $(\mathrm{e}-\mathrm{h}+)$ en procesos tipo Fenton [7].

En el presente trabajo se presenta la síntesis y caracterización de ferritas de cobalto y el estudio de su actividad catalítica en la degradación de diclofenac sódico mediante un proceso tipo foto-Fenton empleando $\mathrm{H}_{2} \mathrm{O}_{2}$ como agente oxidante y radiación Uv-germicida. También se presenta una discusión prelimiar sobre el mecanismo de degradación.

\section{Desarrollo}

Síntesis de materiales: Los materiales se sintetizaron mediante el método de Pechini. Las fuentes de metales empleadas fueron $\mathrm{Co}\left(\mathrm{NO}_{3}\right)_{2} \cdot 6 \mathrm{H}_{2} \mathrm{O}$ y Fe$\left(\mathrm{NO}_{3}\right)_{2} \cdot 6 \mathrm{H}_{2} \mathrm{O}$. Los materiales fueron calcinados en mufla durante 6 horas a 600 y $800^{\circ} \mathrm{C}$ respectivamente.

Caracterización: Por medio de difracción de rayos X (DRX) se analizó la estructura de los materiales obtenidos en un equipo X'Pert Pro-PANalytical, con irradiación $\mathrm{CuK} \alpha, \lambda=1,54 \AA \AA$; en el rango $2 \theta$ de $4^{\circ}$ a $80^{\circ}$. Los picos característicos fueron identificados mediante la base de datos del International Centre of Diffraction Data (ICDD). Los patrones $\mathrm{AB}_{2} \mathrm{O}_{4}(\mathbf{\square}) \mathrm{se}$ corresponden con la ferrita $\mathrm{CoFe}_{2} \mathrm{O}_{4}(00-002-1045)$. Al aumentar la temperatura de calcinación, se observa una mejora en la cristalinidad (Figura 1 (a)).
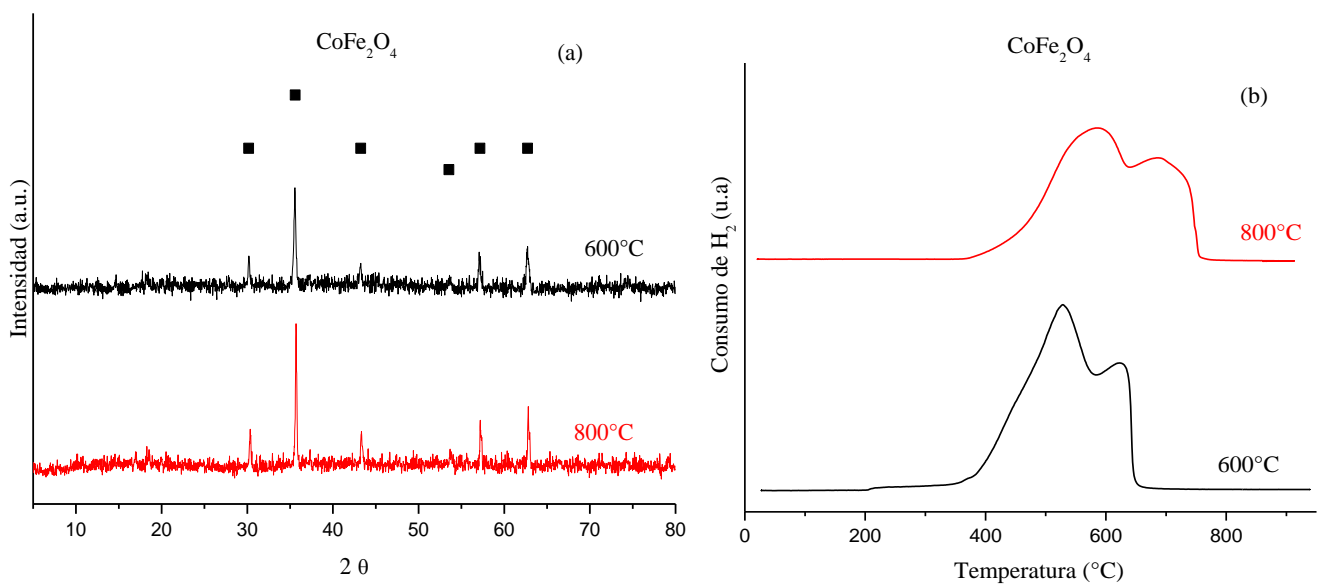

Figura 1.Caracterización de ambos materiales. (a) Patrones de rayos X. (b) Perfiles TPR.

La energía de band gap (EC) de los materiales fue calculada mediante espectroscoía UV-visible con reflectancia difusa, los espectros fueron tomados en un espectrómetro Jasco UV-visible V-650. Los valores obtenidos de la gráfica de (h $\mu *$ 
$\mathrm{KM})^{2}$ vs $(\mathrm{h} \mu)$ [8] fueron: 1.89 y $1.08 \mathrm{eV}$ para $\mathrm{CoFe}_{2} \mathrm{O}_{4}$ calcinada a 600 y $800{ }^{\circ} \mathrm{C}$ respectivamente. La EC en los semiconductores tiene estrecha relación con el tamaño de partícula. Partículas más grandes exhiben una EC más pequeña [9]. Con ello se puede inferir que al aumentar la temperatura de calcinación, aumenta el tamaño de partícula.

La reducción con hidrógeno a temperatura programada (TPR) se realizó en un equipo Micromeritics Chemisorb 2720. Las muestras fueron calentadas a $150^{\circ} \mathrm{C}$ durante 30 minutos en flujo de $\mathrm{N}_{2}$ para remover la humedad adsorbida, luego se aumentó la temperatura a una velocidad de $10 \stackrel{\circ}{\circ} \mathrm{C} / \mathrm{min}$ en presencia de $\mathrm{H}_{2}\left(5 \% \mathrm{H}_{2} / \mathrm{N}_{2}, 20 \mathrm{~mL} / \mathrm{min} \mathrm{STP}\right)$ hasta los $950^{\circ} \mathrm{C}$. La reacción de reducción fue monitoreada mediante el consumo de $\mathrm{H}_{2}$. La primera banda observada en la Figura 1 (b) se puede asignar a la reducción del $\mathrm{Co}^{+2}$ a Co ${ }^{0}$, en conjunto con la del $\mathrm{Fe}^{+3} \mathrm{a} \mathrm{Fe}^{+2}$. La segunda banda, a la reducción del $\mathrm{Fe}^{+2}$ a $\mathrm{Fe}^{0}$. Tonge et al. [10] encontraron que en los perfiles de TPR, con el aumento del tamaño de partícula, los máximos de los picos se corren hacia mayores temperaturas. En ambas muestras se descarta la presencia de $\mathrm{Co}^{+3}$ ya que no presentan bandas a temperaturas inferiores a $260^{\circ} \mathrm{C}[11]$.

El área superficial fue determinada mediante fisisorción de $\mathrm{N}_{2}$ siguiendo el método de Brunauer-Emmett-Teller con un equipo Micromeritics Pulse Chemisorb 2700. Las muestras fueron desgastadas a $390^{\circ} \mathrm{C}$ durante 60 minutos. Las áreas obtenidas fueron 7.4 y $3.7 \mathrm{~m}^{2} / \mathrm{g}$ para el material calcinado a $600^{\circ} \mathrm{C}$ y $800^{\circ} \mathrm{C}$ respectivamente. La disminución en el área superficial podría estar relacionada con el aumento del tamaño de partícula obtenido al calcinar a mayores temperaturas.

La espectroscopia fotoelectrónica de rayos X (XPS) fue medida en un equipo Thermo Scientific K-alpha empleando una fuente monocromática de $\mathrm{Al} \mathrm{K \alpha}(1486.6 \mathrm{eV})$. En el espectro, en la región del Co, la transición 2 $\mathrm{p}_{3 / 2}$ se caracteriza por un pico principal seguido de un satélite a mayores energías, indicando la presencia de $\mathrm{Co}^{+2}$ en la estructura tal como se encontró en el análisis de TPR. Además, la diferencia de energías entre Co $2 \mathrm{p}_{1 / 2}$ - Co $2 \mathrm{p}_{3 / 2}$ es cercana a 15 eV para ambas muestras, lo que confirma la presencia de las especies de cobalto bivalentes [12]. En la región del $\mathrm{Fe}$, el doblete característico $2 \mathrm{p}_{3 / 2}$ y $2 \mathrm{p}_{1 / 2}$ cercano a 711 y $724 \mathrm{eV}$ respectivamente fue deconvolusionado en dos contribuciones. Esto indicaría la presencia de $\mathrm{Fe}^{+3}$ en sitios octaédricos y tetraédricos [13]. Los compuestos tipo espinela siguen la siguiente formula molecular: $\left(\mathrm{Co}_{(1-\lambda)}, \mathrm{Fe}_{(\lambda)}\right)_{\mathrm{A}}\left[\mathrm{Co}_{(\lambda)} \mathrm{Fe}_{(2-\lambda)}\right]_{\mathrm{B}} \mathrm{O}_{4}$ en la cual $[\mathrm{A}]$ y [B] son los sitios tetraédricos y octaédricos respectivamente $\mathrm{y}, \lambda$ es el grado de inversión que indica el $\%$ de $\mathrm{Co}^{+2}$ ocupando sitios octaédricos. De acuerdo con los porcentajes de ocupación de cada sitio obtenidos de la deconvolución de las señales de XPS se encontró que la ferrita calcinada a $600^{\circ} \mathrm{C}$ presenta un $75 \%$ de inversión, mientras que la calcinada a $800^{\circ} \mathrm{C}$ un $83 \%$.

Los espectros de Mössbauer fueron medidos en geometría de transmisión con un espectrómetro de aceleración constante de 512 canales (WissEl, Alemania), utilizando una fuente de ${ }^{57} \mathrm{Co}$ en matriz $\mathrm{Rh}$ de $50 \mathrm{mCi}$ nominal a $25^{\circ} \mathrm{C}$. El espesor ideal de la muestra se evaluó considerando los porcentajes en peso de los diferentes elementos de cada sólido (se usaron aproximadamente $100 \mathrm{mg}$ de polvo para un soporte de 1,8 cm de diámetro). La calibración de velocidad se realizó utilizando una lámina de $\alpha$-Fe de $12 \mu \mathrm{m}$ de espesor como referencia. Todos los espectros se recogieron a $25^{\circ} \mathrm{C}$. Cada espectro se plegó para minimizar los efectos geométricos y los datos experimentales se ajustaron utilizando un programa comercial con restricciones denominado Recoil20.

Ambos materiales exhibieron un sextuplete ampliado con hombros de 5 o 6 picos hacia velocidades más altas. Esta descripción cualitativa indica que varios sitios de hierro están presentes en estas ferritas. Para explicar la existencia de estos diferentes sitios, se debe considerar el súper intercambio magnético entre dos cationes vecinos más próximos a través de un anión no magnético $\left(\mathrm{O}^{-2}\right)$. Estas interacciones modifican los momentos magnéticos iónicos, que son proporcionales a los campos magnéticos hiperfinos, detectados por la espectroscopia de Mössbauer. De las diferentes interacciones de súper intercambio, las que ocurren entre $\mathrm{Fe}^{3+}$ ubicado en los sitios $[\mathrm{A}]$ y $[\mathrm{B}]$ son las más fuertes. Esta clase de interacción se llama A-B. Por otro lado, las interacciones A-A y B-B tienen mucha menos intensidad.

En $\mathrm{CoFe}_{2} \mathrm{O}_{4}$ cada $\mathrm{Fe}^{3+}[\mathrm{A}]$ se encuentra rodeado por doce $\mathrm{Fe}^{3+}$ [B]. Por lo tanto, el reemplazo de uno de estos iones [B] por un $\mathrm{Co}^{2+}$ (de menor momento magnético que un ión $\mathrm{Fe}^{3+}$ ) no producirá un porcentaje de cambio importante en la interacción de super-intercambio y el campo magnético hiperfino de los iones $\mathrm{Fe}^{3+}[\mathrm{A}]$ no se verá alterado significativamente por el número de $\mathrm{Co}^{2+}$ vecinos.

Por el contrario, los $\mathrm{Fe}^{3+}[\mathrm{B}]$ sólo poseen seis $\mathrm{Fe}^{3+}[\mathrm{A}]$ como vecinos más próximos. Por esta razón, si un $\mathrm{Fe}^{3+}[\mathrm{A}]$ es reemplazado por un $\mathrm{Co}^{2+}$ la interacción de super-intercambio se reducirá en un porcentaje importante al igual que el correspondiente campo hiperfino magnético.

Teniendo en cuenta estas consideraciones el espectro Mössbauer de las muestras fue ajustado siguiendo la metodología propuesta por Sawatzky et al. [14], [15] y por Ferreira et al. [16]. Existe una excelente concordancia entre los parámetros hiperfinos magnéticos obtenidos del ajuste utilizando este modelo y aquellos reportados por Ferreira et al [16]. La ferrita calcinada a $600^{\circ} \mathrm{C}$ presenta una estructura con un $74 \%$ de inversión mientras que el material calcinado a $800^{\circ} \mathrm{C}$ un $72 \%$.

Mediante la espectroscopia Mössbauer y el XPS se encontraron estructuras similares a las reportadas previamente, 
espinelas de Fe-Co con inversión parcial de entre el 70 y $80 \%$ [17]. Las estructuras encontradas por ambas técnicas coinciden. Las diferencias se pueden atribuir a que el XPS es una técnica superficial mientras que la espectroscopia Mössbauer da información del seno del material.

Evaluación catalítica: La reacción de degradación de diclofenac sódico se llevó a cabo a $25^{\circ} \mathrm{C}$ en un reactor tubular mediante un proceso foto-Fenton. Las muestras fueron identificadas como Mx dónde x representa el tiempo al que fue tomada: 0, 15, 60, 180 y 240 minutos de reacción. El seguimiento del diclofenac se llevó a cabo a través de cromatografía líquida de alta resolución (HPLC) en un equipo Perkin Elmer Serie 2020 con columna Agilent Zorbax Eclipse Plus C18 4.6x250mm y detector Uv-Vis y a través de espectroscopia Uv-vis en un equipo Perse T7DS. La remoción de carbono orgánico total (TOC) fue determinada con un equipo Shimadzu TOC-L CSN. La reducción del TOC fue calculada con la siguiente ecuación: [(TOC $\left.\mathrm{T}_{\mathrm{M}}-\mathrm{TOC}_{\mathrm{M} 240}\right)$ x100] / $\mathrm{TOC}_{\mathrm{M} 0}$. El consumo de $\mathrm{H}_{2} \mathrm{O}_{2}$ fue evaluado mediante titulación iodométrica[18].

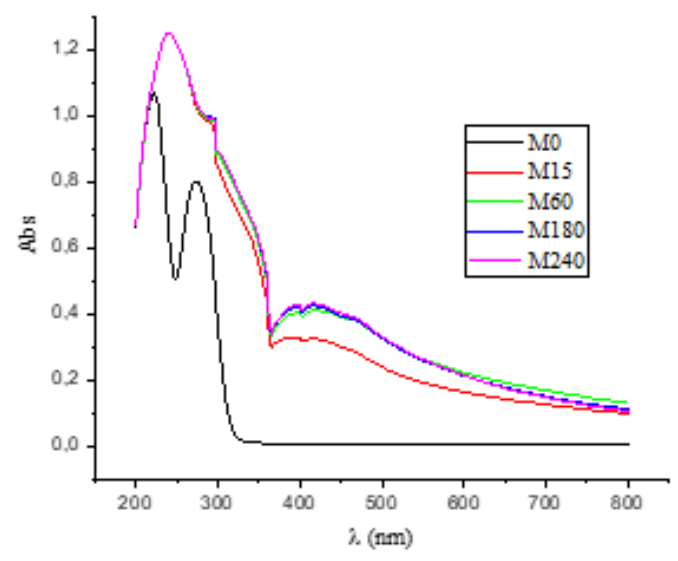

Figura 2. Fotólisis

El espectro Uv-vis de la molécula de diclofenac presenta dos máximos a 221 y $272 \mathrm{~nm}$ respectivamente (Figura 2 [M]). Luego de una corta exposición a luz Uv (Fotólisis) aparece un nuevo máximo a $240 \mathrm{~nm}$ (Figure 2 [M $\left.\mathrm{M}_{15}\right]$ ). De acuerdo con lo encontrado por Iovino et al.[19] este nuevo pico es asociado a la aparición del dímero carbazol, un intermediario muy estable y de color amarillento. De esto se puede inferir que luego de 15 minutos de acción de luz, el diclofenac sódico es transformado en carbazol; mediante HPLC fue determinado que la concentración de diclofenac fue menor a $2 \mathrm{mg} / \mathrm{L}$. En esta etapa es importante destacar que no hubo mineralización.

Con el objeto de evaluar la incidencia de la ferrita sintetizada se probaron diferentes blancos de reacción. Para los siguientes casos no se obtuvo degradación en ausencia de radiación: Adsorción, Proceso tipo Fenton (catalizador y peróxido) y la solución de diclofenac en contacto con $\mathrm{H}_{2} \mathrm{O}_{2}\left(\mathrm{H}_{2} \mathrm{O}_{2}\right)$. Cuando al proceso de fotólisis se le suma la presencia de catalizador, se obtiene degradación del diclofenac, pero no de los intermediarios de degradación. Finalmente, con la presencia de radiación y agente oxidante se obtuvo degradación del diclofnac y una mineralización del 52\% (Tabla 1).

\begin{tabular}{lcccccc}
\hline & Ads & Fotólisis & $\mathrm{H}_{2} \mathrm{O}_{2}$ & Fenton & Luz y Cat & $\mathrm{Luz} \mathrm{y} \mathrm{H}_{2} \mathrm{O}_{2}$ \\
\hline \% TOC removido \pm Error & $0.93 \pm 0.01$ & $0.97 \pm 0.01$ & $0.85 \pm 0.01$ & $2.30 \pm 0.01$ & $3.21 \pm 0.01$ & $51.89 \pm 0.01$ \\
\hline
\end{tabular}

Tabla 1. Porcentaje de remoción de TOC en los blancos de reacción.

Con el objeto de optimizar las condiciones de reacción, se varió el volumen de oxidante agregado y la masa de catalizador. El volumen de $\mathrm{H}_{2} \mathrm{O}_{2}$ se modificó entre 0.6 y $1.8 \mathrm{~mL} / \mathrm{L}$. Con la concentración de $1.2 \mathrm{~mL} / \mathrm{L}$ se obtuvieron los mejores resultados en relación a la mineralización de los contaminantes y el consumo de $\mathrm{H}_{2} \mathrm{O}_{2}$ (Tabla 2). La masa de catalizador se varió entre $0.2 \mathrm{~g} / \mathrm{L}$ a $1 \mathrm{~g} / \mathrm{L}$. Como no se observaron diferencias significativas, se trabajó con la menor concentración.

Luego de optimizar las condiciones de reacción ( $40 \mathrm{mg} / \mathrm{L}$ de diclofenac sódico, $0.2 \mathrm{~g} / \mathrm{L}$ de catalizador y $1.2 \mathrm{~mL} / \mathrm{L}$ de $\mathrm{H}_{2} \mathrm{O}_{2}$, bajo radiación Uv) ambos materiales fueron testeados. (Figura 3). Con ambos materiales se logró la degradación completa de la molécula de diclofenac. Sin embargo, se observan diferentes velocidades de descomposición, en este sentido el catalizador calcinado a $800^{\circ} \mathrm{C}$ es más rápido en la degradación de los subproductos obtenidos. Sumado a esto, 
con dicho material se logra una mayor mineralización del TOC, del orden del $86 \%$ vs $75 \%$ para el material calcinado a $600^{\circ} \mathrm{C}$.

\begin{tabular}{cccc}
\hline & $\mathbf{0 . 6}(\mathbf{m L} / \mathrm{L})$ & $\mathbf{1 . 2}(\mathbf{m L} / \mathrm{L})$ & $\mathbf{1 . 8}(\mathbf{m L} / \mathrm{L})$ \\
\hline$\%$ TOC removido \pm Error & $59.61 \pm 0.02$ & $85.92 \pm 0.02$ & $83.60 \pm 0.01$ \\
$\% \mathrm{H}_{2} \mathrm{O}_{2}$ consumido \pm Error & $91 \pm 3$ & $91 \pm 3$ & $90 \pm 3$ \\
\hline
\end{tabular}

Tabla 2. Resultados cobtenidos con la variación del volumen de $\mathrm{H}_{2} \mathrm{O}_{2}$ agregado.
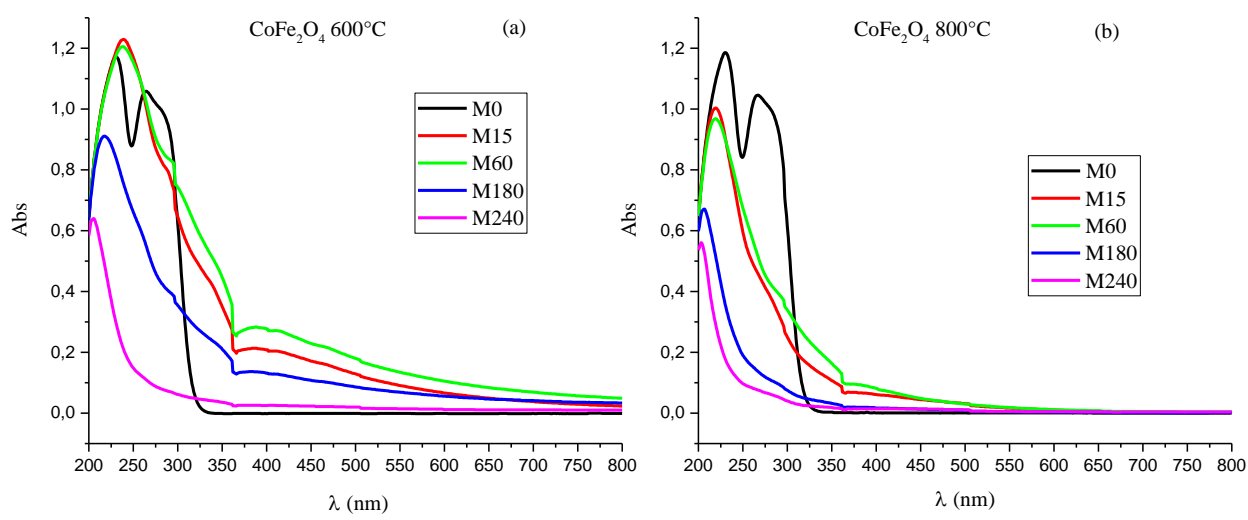

Figura 3. Variación del catalizdor. (a) Ferrita calcinada a $600^{\circ} \mathrm{C}$. (b) Ferrita calcinada a $800^{\circ} \mathrm{C}$

La mayor actividad catalítica obtenida con el material calcinado a $800^{\circ} \mathrm{C}$ puede atribuirse a su menor EC lo cuál promueve más fácilmente la formación del par electrón-hueco[20]. Además, esto puede deberse a la mayor presencia superficial de $\mathrm{Co}^{+2}$ en sitios octaédricos. La transferencia de carga en las ferritas es descripta por el mecanismo de hopping electrónico entre los sitios B de la red. La oxidación de $\mathrm{Co}^{+2}$ a $\mathrm{Co}^{+3}$ debe ser compensada con la reducción del $\mathrm{Fe}^{+3}$ to $\mathrm{Fe}^{+2}$ o la migración de $\mathrm{Co}^{+2}$ a sitios tetraédricos [13].

En estas condiciones se evaluó la reutilización del catalizador en tres ciclos consecutivos. El material fue separado del medio de reacción mediante filtración y secado en estufa a $50^{\circ} \mathrm{C}$. Los resultados no mostraron pérdida de actividad obteniéndose la degradación completa del contaminante y una remoción de TOC del 86\%, 84\% y 84\% en cada ciclo respectivamente.

Mecanismo de reacción: Los intermediarios de degradación fueron identificados a través de cromatografía gaseosa con espectrofotometría de masas (GC-MS) en un equipo Perkin Elmer Clarus 560 equipado con una columna Alltech Econo CAP-EC-WAX. Debido a las características del equipo solo pudieron identificarse intermediarios de bajo peso molecular, entre ellos: 2,4 diclorofenol, 2 cloro-1,2 difenil etanona, acetamida y pequeñas moléculas ácidas como ácido oxálico y acético. De esto se puede inferir que la reacción de degradación sigue un mecanismo tipo radicalario. Para confirmarlo, se llevó a cabo una reacción en la cual se le agregaron $15 \mathrm{~mL}$ de metanol al medio de reacción no se observó degradación de los intermediarios, ni mineralización. Esto se debe a que alcoholes como el etanol o el metanol actúan como captores de radicales $* 0 H$ haciendo más lenta la reacción[21].

\section{Conclusiones}

Se obtuvieron fases puras de ferritas de cobalto-hierro mediante el método de Pechini calcinados a 600 y $800^{\circ} \mathrm{C}$. Ambos materiales tuvieron estructura de espinela con inversión parcial entre el 70 y $80 \%$. El aumento de la temperatura de calcinación mejoró la cristalinidad de las muestras y aumentó el tamaño de partícula.

Los materiales sintetizados mostraron buenas propiedades catalíticas en la degradación de diclofenac sódico en un proceso tipo foto-Fenton bajo radiación Uv germicida. Con la ferrita calcinada a $800^{\circ} \mathrm{C}$ se obtuvo la degradación completa del contaminante y $86 \%$ de mineralización del TOC. Además, mostró excelentes resultados en tres reúsos consecutivos. La mayor actividad catalítica de este material es atribuible a su menor EC. Se confirmó que el proceso de degradación sigue un mecanismo radicalario. 


\section{Referencias}

[1] V. Geissen et al., "Emerging pollutants in the environment: A challenge for water resource management," Int. Soil Water Conserv. Res., vol. 3, no. 1, pp. 57-65, 2015, doi: 10.1016/j.iswcr.2015.03.002.

[2] D. Simazaki, R. Kubota, T. Suzuki, and M. Akiba, "ScienceDirect Occurrence of selected pharmaceuticals at drinking water purification plants in Japan and implications for human health," Water Res., vol. 76, pp. 187-200, 2015, doi: 10.1016/j.watres.2015.02.059.

[3] M. E. Valdés, M. V. Amé, M. de los A. Bistoni, and D. A. Wunderlin, "Occurrence and bioaccumulation of pharmaceuticals in a fish species inhabiting the Suquía River basin (Córdoba, Argentina)," Sci. Total Environ., vol. 472, pp. 389-396, 2014, doi: 10.1016/j.scitotenv.2013.10.124.

[4] A. Achilleos, E. Hapeshi, N. P. Xekoukoulotakis, D. Mantzavinos, and D. Fatta-Kassinos, "Factors affecting diclofenac decomposition in water by UV-A/TiO2 photocatalysis," Chem. Eng. J., vol. 161, no. 1-2, pp. 53-59, 2010, doi: 10.1016/j.cej.2010.04.020.

[5] J. L. Wang and L. J. Xu, "Advanced oxidation processes for wastewater treatment: Formation of hydroxyl radical and application," Crit. Rev. Environ. Sci. Technol., vol. 42, no. 3, pp. 251-325, 2012, doi: 10.1080/10643389.2010.507698.

[6] F. Zhang, C. Wei, K. Wu, H. Zhou, Y. Hu, and S. Preis, "Mechanistic evaluation of ferrite AFe2O4 (A = Co, Ni, $\mathrm{Cu}$, and $\mathrm{Zn}$ ) catalytic performance in oxalic acid ozonation," Appl. Catal. A Gen., vol. 547, no. February 2018, pp. 60-68, 2017, doi: 10.1016/j.apcata.2017.08.025.

[7] E. M. Casbeer, V. K. Sharma, Z. Zajickova, and D. D. Dionysiou, "Kinetics and mechanism of oxidation of tryptophan by ferrate(VI)," Environ. Sci. Technol., vol. 47, no. 9, pp. 4572-4580, 2013, doi: 10.1021/es305283k.

[8] A. B. Murphy, "Band-gap determination from diffuse reflectance measurements of semiconductor films, and application to photoelectrochemical water-splitting," Sol. Energy Mater. Sol. Cells, vol. 91, no. 14, pp. 1326-1337, 2007, doi: 10.1016/j.solmat.2007.05.005.

[9] M. M. Karkare, "Estimation of Band Gap and Particle size of TiO2 nanoparticle synthesized using Sol gel technique," Proc. - 2014 IEEE Int. Conf. Adv. Commun. Comput. Technol. ICACACT 2014, no. Iv, pp. 2-6, 2015, doi: 10.1109/EIC.2015.7230747.

[10] K. H. Tonge, "Particle size effects in temperature programmed topochemical reactions," Thermochim. Acta, vol. 74, no. 1-3, pp. 151-166, 1984, doi: 10.1016/0040-6031(84)80015-5.

[11] U. A. Agú, M. I. Oliva, S. G. Marchetti, A. C. Heredia, S. G. Casuscelli, and M. E. Crivello, "Synthesis and characterization of a mixture of $\mathrm{CoFe} 2 \mathrm{O} 4$ and $\mathrm{MgFe} 2 \mathrm{O} 4$ from layered double hydroxides: Band gap energy and magnetic responses," J. Magn. Magn. Mater., vol. 369, pp. 249-259, 2014, doi: 10.1016/j.jmmm.2014.06.046.

[12] L. Lonappan, S. K. Brar, R. K. Das, M. Verma, and R. Y. Surampalli, "Diclofenac and its transformation products: Environmental occurrence and toxicity - A review," Environ. Int., vol. 96, pp. 127-138, 2016, doi: 10.1016/j.envint.2016.09.014.

[13] Z. Zhou et al., "Electronic structure studies of the spinel CoFe $2 \mathrm{O} 4$ by X-ray photoelectron spectroscopy," Appl. Surf. Sci., vol. 254, no. 21, pp. 6972-6975, 2008, doi: 10.1016/j.apsusc.2008.05.067.

[14] G. A. Sawatzky, F. Van Der Woude, and A. H. Morrish, "Cation distributions in octahedral and tetrahedral sites of the ferrimagnetic spinel CoFe2O4," J. Appl. Phys., vol. 39, no. 2, pp. 1204-1205, 1968, doi: 10.1063/1.1656224.

[15] G. A. Sawatzky, F. Van Der Woude, and A. H. Morrish, "Mössbauer study of several ferrimagnetic spinels," Phys. Rev., vol. 187, no. 2, pp. 747-757, 1969, doi: 10.1103/PhysRev.187.747.

[16] T. A. S. Ferreira, J. C. Waerenborgh, M. H. R. M. Mendonça, M. R. Nunes, and F. M. Costa, "Structural and morphological characterization of FeCo2O4 and CoFe2O4 spinels prepared by a coprecipitation method," Solid State Sci., vol. 5, no. 2, pp. 383-392, 2003, doi: 10.1016/S1293-2558(03)00011-6.

[17] B. S. Holinsworth et al., "Chemical tuning of the optical band gap in spinel ferrites: CoFe $2 \mathrm{O} 4$ vs NiFe2O4," Appl. Phys. Lett., vol. 103, no. 8, pp. 2-5, 2013, doi: 10.1063/1.4818315.

[18] T. W. Davis, "Fast Neutron and Gamma Radiation '," vol. 130, no. 2, 1952.

[19] P. Iovino, S. Chianese, S. Canzano, M. Prisciandaro, and D. Musmarra, "Photodegradation of diclofenac in wastewaters," Desalin. Water Treat., vol. 61, no. 2, pp. 293-297, 2017, doi: 10.5004/dwt.2016.11063.

[20] T. P. Hough, Ed., Recent Developments in Solar Energy, 1st ed. New York: Nova Science Publishers Inc., 2007.

[21] N. L. Finčur et al., "Removal of alprazolam from aqueous solutions by heterogeneous photocatalysis: Influencing factors, intermediates, and products," Chem. Eng. J., vol. 307, pp. 1105-1115, 2017, doi: 0.1016/j.cej.2016.09.008. 